\title{
A WIDEBAND THERMO-ACOUSTIC SOUND GENERATOR ON NANOPOROUS POLYMERIC SUBSTRATE
}

\author{
A. Kim, G. Chitnis, S. H. Song, and B. Ziaie* \\ Birck Nanotechnology Center, Purdue University, USA
}

\section{INTRODUCTION}

Ultrasonic transducers operating in air are important components in systems used for ranging, surveillance, object identification, and air flow measurement [1]. In ranging and object identification, a wideband transducer is needed in order to achieve a better axial resolution. Some of the most commonly used ultrasonic sources are inherently resonant and hence narrowband. In case of thermo-acoustic sound generator (thermophone) electrical energy is converted to sound waves through Joule heating of a resistor without any mechanical vibration, thus allowing for a wideband operation [2]. Recently, there has been a renewed interest in this mode of sound generation. These efforts include various structures consisting of a thin conductor (e.g., aluminum, carbon nanotubes, ITO, and grapheme) deposited on thermally insulating substrates such as porous silicon, polyethylene-terephthalate, and paper [3-6]. Many of these methods rely on complicated material and fabrication processes, which are not compatible with standard CMOS and other common microfabrication techniques. Here, we report on a thermophone using a thin metallic layer deposited on a nanoporous polymeric substrate. The fabrication method is simple, low-temperature, and compatible with active electronics.

\section{DEVICE STRUCTURE AND FABRICAION}

The thermophone (Figure 1) consists of a mechanical support (silicon, glass, etc.) coated with a thermally insulating nanoporous polymeric layer covered by an electrically conductive thin metal layer. When current is passed through the resistor, temperature of the metal rises due to Joule's heating, thus heating the surrounding air. Since the thin metal layer has very low heat capacity, when excited with alternating current (frequency f), it results in rapid variation of temperature leading to expansion and contraction of surrounding air at doubled frequency $(2 \mathrm{f})$, generating sound waves.

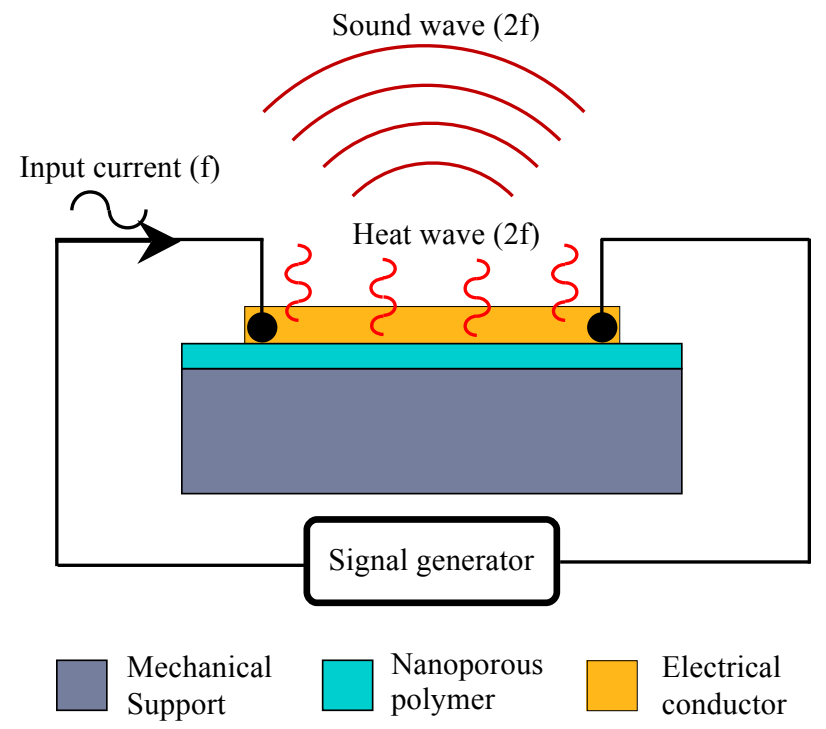

Figure 1: Schematic of the thermo-acoustic ultrasonic sound generator

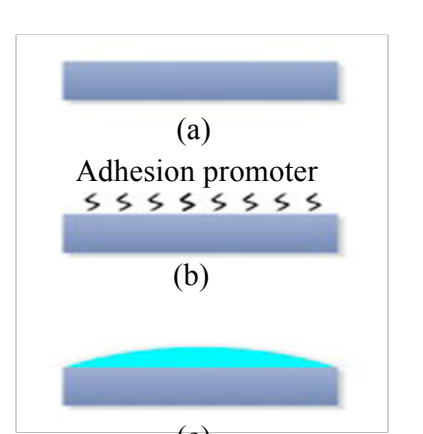

(c)

Glass (pyrex 7740)

Hydrogel

Figure 2: Fabrication process

The performance of thermophone is highly dependent on the properties of thermal insulating layer. This layer helps in efficient transfer of heat to the air. A nano-material with adjustable porosity (and thus thermal conductivity) yields a better control over device performance.

Figure 2 shows the fabrication process. For our experiments, we used glass substrate and air-dried hydrogel [Poly(mAA-coAAm)] as the nanoporous layer [7]. One can easily control the porosity of the gel by adjusting the ratio of cross-linker of its precursors. Although air-drying leads to collapse of porous structure, some porosity is expected to be retained (complete retention of porosity can be achieved through critical point drying). The thickness of the hydrogel can be controlled by adjusting the weight applied while curing the gel (a weight of $9 \mathrm{~g}$ resulted in 14 $\mu \mathrm{m}$ thick gel after drying). Finally, a thin layer of gold $(40 \mathrm{~nm})$ was patterned by shadow masking. Figure 3 shows photograph of a fabricated device.

\section{RESULTS AND DISCUSSION}

The hydrogel based thermophone was characterized in an anechoic room using a high-frequency condenser microphone. Figure 4 depicts the experimental set up. A sinusoidal voltage signal $\left(4.5 \mathrm{~V}_{\mathrm{pp}}\right)$ was applied across the serpentine resistor and generated acoustic signal was recorded at a distance of $30 \mathrm{~mm}$.

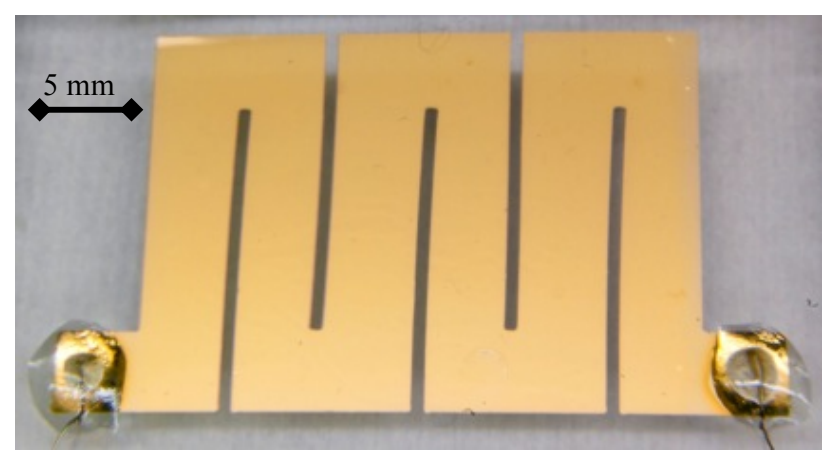

Figure 3: Optical photograph of fabricated prototype 


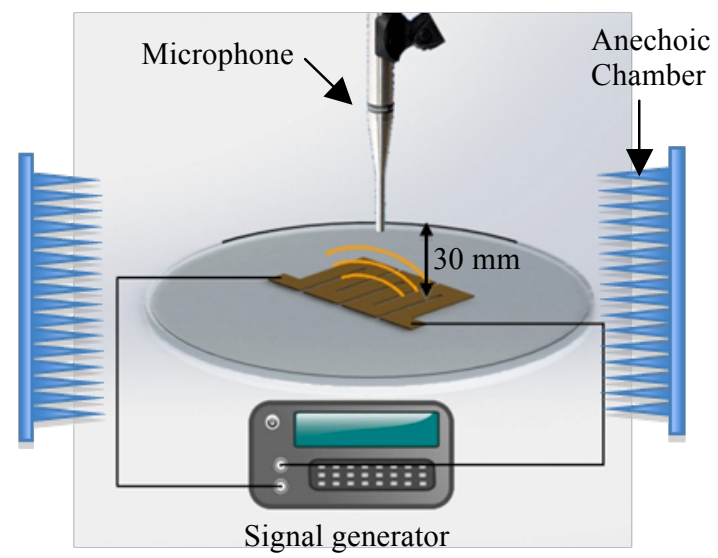

Figure 4: Schematic of experimental set up

Figure 5(a) shows input and output signal on the same plot with the output sound wave double the input signal frequency. Figure 5(b) shows sound pressure level (SPL) obtained at various frequencies. Input power was kept constant at $0.511 \mathrm{~W}$ for all the measurements. The performance of the device remains consistent up to $80 \mathrm{kHz}$ (limited by the microphone used in the experiment).

It is not straightforward to compare the efficiency of our device with other acoustic devices due to difference in powering mechanism. Output SPL of our device is proportional to input power, while that of piezoelectric or electrostatic based acoustic devices is proportional to input voltage. In case of electromagnetic micro-speaker [8], SPL of $93 \mathrm{~dB}$ at $5 \mathrm{kHz}$ has been reported at input voltage of $1.5 \mathrm{~V}$ (320 $\mathrm{mW}$ power). The piezoelectric micromachined ultrasonic transponder shows a peak SPL of $85 \mathrm{~dB}$ at 0.4 $\mathrm{V}$ and $214 \mathrm{kHz}$ [9]. Unlike thermophone, electromagnetic and piezoelectric based devices, which rely on mechanical vibration,

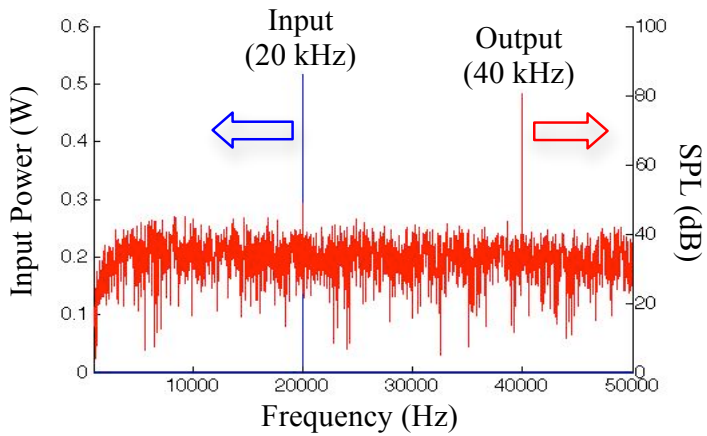

(a)

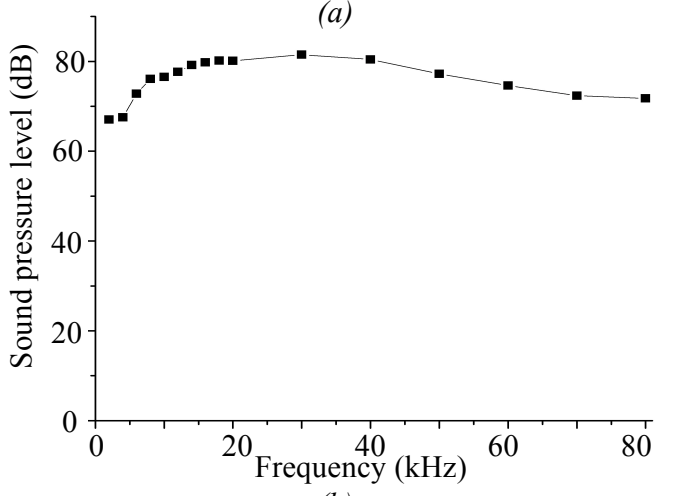

(b)

Figure 5. (a) SPL and Input power relations. Input at $20 \mathrm{kHz}$ generates output at $40 \mathrm{kHz}$, which double the frequency, (b) Frequency response of $S P L$. show a peak performance near the resonance but output signal attenuates before and after the resonant frequency. The performance of thermophone consistent over at least $80 \mathrm{kHz}$ is a major advantage over these conventional ultrasonic transponders used for imaging or in-air ranging applications. Further, the structure and fabrication process presented here is simple enough to be adapted for miniaturization of the device. Although frequency doubling behavior of thermophone is somewhat inconvenient for audible speaker application, one can easily circumvent the problem by using an electronic circuit that reduces input frequency to half before the input [10].

\section{CONCLUSIONS}

We presented a simple, low-temperature method for fabricating a wide-band $(>80 \mathrm{kHz})$ thermo-acoustic sound generator on a nanoporous polymeric substrate. We were able to achieve up to $80 \mathrm{~dB}$ of sound pressure level with input power of only $0.511 \mathrm{~W}$. One can easily control the parameters such as gel thickness, porosity, and metal thickness to further improve the performance. Wide-band ultrasonic performance, simplicity of structure and scalability of the fabrication process make this device suitable for a wide range of ranging and imaging applications.

\section{REFERENCES}

[1] W. Manthey, N. Kroemer, and V. Magori, "Ultrasonic transducers and transducer arrays for applications in air," Measurement Science and Technology, vol. 3, no. 3, pp. 249261, Mar. 1992.

[2] H. D. Arnold and I. B. Crandall, "The thermophone as a precision source of sound," Physical Review, 1917.

[3] H. Shinoda, T. Nakajima, K. Ueno, and N. Koshida, "letters to nature Thermally induced ultrasonic emission from porous silicon," Reactions, vol. 400, no. August, pp. 853-855, 1999.

[4] L. Xiao et al., "Flexible, stretchable, transparent carbon nanotube thin film loudspeakers.," Nano letters, vol. 8, no. 12, pp. 4539-45, Dec. 2008.

[5] H. Tian et al.,"Poly(3,4-ethylenedioxythiophene)poly(styrenesulfonate)-based organic, ultrathin, and transparent soundemitting device," Applied Physics Letters, vol. 99, no. 23, p. 233503, 2011.

[6] H. Tian et al., "Transparent, flexible, ultrathin sound source devices using Indium Tin oxide films," Applied Physics Letters, vol. 99, no. 4, p. 043503, 2011.

[7] Z. Ding, A. Salim, and B. Ziaie, "Squeeze-film hydrogel deposition and dry micropatterning.," Analytical chemistry, vol. 82, no. 8, pp. 3377-82, Apr. 2010.

[8] M. C. Cheng, W. S. Huang, and S. R. S. Huang, "A silicon microspeaker for hearing instruments.," Journal of Micromechanics and Microengineering, vol. 14, no. 7, pp. 859-866. May. 2004.

[9] R. J. Przybyla, S. E. Shelton, A. Guedes, I. I. Izyumin, M. H. Kline, D. A. Horsley and B. E. Boser, "In-Air Rangefinding With an AlN Piezoelectric Micromachined Ultrasound Transducer.," IEEE Sensors Journal, vol. 11, no 11, pp. 2690-2697. Nov. 2011.

[10] R. W. J. Barker, K. P. Corrin, "Sinusoidal-FrequencyHalving Technique.," Electronics Letters, vol. 13, no 7, pp. 201-202, Mar. 1977.

\section{ACKNOWLEDGEMENTS:}

Authors would like to thank Andy Jessop and Prof. Bolton for their help with the acoustic measurements.

\section{CONTACT:}

*B. Ziaie, tel: +1-765-494-0725; bziaie@purdue.edu 